\title{
Comorbidity or consanguinity
}

\author{
PETER TYRER
}

It is a matter for concern when a subject becomes discussed widely in clinical research but does not have anything like the same impact on clinical practice. This could be because good research with clinical implications will always antedate clinical practice but it could also indicate that the researchers are losing contact with their clinical base. The subject of comorbidity is a good example. Comorbidity appears repeatedly in the titles of articles published in psychiatric journals particularly in the North American sub-continent, and is becoming increasingly familiar to epidemiologists and social scientists as well as psychiatrists. However, if the subject is brought into clinical discussions in most parts of the world there is likely to be a slightly embarrassed silence before moving on as if the word had never existed. This can be for many reasons, including ignorance of the word in any of its five meanings that Hermann van Praag discusses, indifference to its clinical implications or consciously regarded as irrelevant to decision making in practice.

As one looks into the pages of the supplement with this issue one wonders how comorbidity can be irrelevant because it is clearly a very significant clinical predictor. However, one also needs to be clear exactly what the word means. It has been defined most convincingly by Feinstein as "any distinct additional clinical entity that has existed or that may occur during the clinical course of a patient who has the index disease under study" (Feinstein, 1970). The use of the term in current psychiatric practice is not quite as Feinstein envisaged. Nowadays, particularly in those countries in which DSMIV (American Psychiatric Association, 1994) rules the classification of mental disorders, comorbidity is regarded as the simultaneous presence of two or more DSM-IV diagnoses. This often includes two or more diagnoses from both the same axis of the classification as well as those from different axes (e.g. mental state and personality disorder, physical disorder and mental state disorder).

The major problem is that what is true comorbidity (separate diseases) and what is false comorbidity (consanguinity, or such an intimate relationship between the disorders that they are one and the same) is difficult to determine and certainly can not be confirmed in simple crosssectional studies. Thus, for example, Professor Maj's finding that depression is a common feature in those who have HIV infection is understandable in view of the prognosis of HIV disease, and the evidence that the depression is more severe if the onset of major symptoms is sudden, also reinforces the notion that the depression is a direct consequence of the conscious awareness of the diagnosis and its implications (i.e. a consanguinous diagnosis) (Fell et al, 1993). At the same time, however, we also have abundant evidence that HIV infection affects the brain and can lead to a range of psychological disturbances covered by the description 'HIV encephalopathy' and 80 it is possible that at least some cases of depression are secondary to viral involvement of the central nervous system (Fernandez, 1989).

In other instances it is much easier to argue that conditions are truly co-morbid. The various disorders of the thyroid and adrenal axis described by Musselman and Nemeroff are often associated with psychiatric disturbance and although this is again predictable in view of the importance of the pituitary in much of mental functioning, there is a good case for making out that the mental and physical conditions are relatively independent as one is not a direct consequence of the other. The association between pain and affective disturbance, particularly depression, is also possible to fit into a co-morbid model. Although it is natural to become depressed after repeated pain from whatever cause the relationship between them is not a simple cause and effect one as Van Korff and Simon describe in this supplement. Pain is not only associated with depression but also frequently with anxiety and the extent to which the affective symptoms interrelate often has considerable bearing on the expression of the pain. Similarly the relationship between depression and various types of cancer is also difficult to interpret but now there is abundant evidence that affective symptoms can occur before conscious knowledge of the diagnosis of cancer it is reasonable to argue, as Spiegel has done in his contribution to this supplement, that depressive disorders are specifically associated with some 
forms of cancer and are not adjustment reactions to its diagnosis.

It is when one gets into the area of pure psychiatric disorders that co-morbidity increasingly looks like consanguinity. One of the reasons that clinicians do not immediately get attracted to the idea of co-morbidity is that they ignore, or more accurately allow for, the phenomenon in their clinical diagnoses. The good clinician in psychiatry is able to get to the heart of a problem when many different strands are presented on the surface. When these are directly related to the main one or consequences of it there is little point concentrating on the sideshows and they tend to be ignored. This perhaps is hinted at by Norman Sartorius' finding that research workers using the new ICD-10 classification (World Health Organization, 1992) identify many more diagnoses than clinicians examining the same clinical material. Although one interpretation of this is that ICD-10 diagnoses may be set at too low a threshold (and there is evidence from some other sources that this might be true), it is still equally possible that the diagnoses identified in the formal ICD-10 assessment would be dismissed as clinically unimportant by the practising clinician. Dr Sartorius' finding that the ICD prevalence figures are twice as high as the diagnoses given by clinicians is a matter of some concern.

One possible explanation for the great interest in comorbidity is given by David Goldberg in discussing dimensional models of mental illness. Clearly if such illness is best perceived as a dimensional continuum, apparent co-morbidity of categorical diagnoses may be extremely high although its clinical significance less so. Those who believe that categorical diagnoses are useful, and it is probably fair to say that such people are in the ascendant at present in view of the recent expansion of categories in both the ICD and DSM systems, can easily find many more diagnoses by rummaging about the lower echelons in the dimensional system. However, we need to remind ourselves that all the diagnoses currently used are merely working hypotheses and should not be regarded as sacrosanct. Unfortunately, as Goldberg rightly says, there is a tendency for those who use categorical diagnoses frequently to come to believe in them and then act as champions for them against contrary evidence.

The most prominent area of co-morbidity in mental disorders is between depression and anxiety. This is a very important issue to resolve, not least because depression and anxiety are now in different major sections of both ICD-10 and DSM-IV. When $58 \%$ of those in surveys such as that reported by Kessler and his colleagues with major depressive disorder also have a diagnosed life-time anxiety disorder it is reasonable to ask whether the two might be part of the same condition. Brown and his colleagues suggest that the co-morbidity might be explained by common risk factors in childhood and Kendler and Andrews, approaching the subject from slightly from slightly different angles, conclude that genetic factors may also account for this high level of co-morbidity. Andrews suggests that personality vulnerability may account for the tendency to get both anxiety and depressive disorders and provides genetic evidence for this hypothesis. Jules Angst also provides convincing evidence from his extensive cohort studies that anxiety is closely linked with depression and in the brief form of the disorder are even more commonly associated. Evidence is now accumulating that in minor forms of depression and anxiety the two conditions are almost inseparable (Tyrer, 1992) whereas in the more severe ones either the anxiety (usually in the form of panic) or the depression seems to take precedence and it may be possible to regard one sometimes as the major disorder (Breier et al, 1984). The importance of personality status as an intervening variable in provoking vulnerability to both moods is also now becoming appreciated. Despite our best attempts we have been unable to find a natural point at which mild mixed anxietydepression becomes severe depression (with either psychotic symptoms or a time course including mania) and until we resolve this issue our classification of a major group of disorders will tend to look somewhat confused to an outside observer.

Van Praag brings clinicians and researchers into line in his conclusion that the notion of comorbidity 'conceals more than it clarifies'. It is a concept that brings together the exciting and interesting contributions in this supplement but it does little more than describe associations; it does not tell us much about their generation and implications. Van Praag gives five interesting and challenging interpretations of co-morbidity and it is interesting that he regards his last interpretation, a return to the notion of diagnosis as a reaction form in the Meyerian tradition, as the most attractive of these. If Van Praag is right this would mean that much of our current classification is flawed. Certainly there are many who would like to abandon the totally atheoretical classification that is typical of both ICD-10 and DSM-IV. Empiricism has been a good bricklayer in psychiatric classifications but without direction it is in danger of only making walls. 


\section{Roferences}

American Psychiatric Association (1994) Diagmastic and Statistical Marual of Mental Disorders (Fourth edn) (DSMIV). Washington, DC: APA.

Breirr, A., Charney, D. S. \& Hennnger, G. R. (1984) Major depression in patients with agoraphobia and panic disorder. Archtves of General Pyschiatry, 41, 1129-1135.

Fenssten, A. (1970) The pre-therapeutic classification of comorbidity in chronic disease. Journal of Chronic Diseases, 23, 455-462.
Feul, M., Newman, S., Herres, M., et al (1993) Mood and peychiatric disturbance in HIV and AIDS: changes over time. British Journal of Psychiatry, 162, 604-610.

FERNANDEZ, F. (1989) Anxiety and the neuropoychiatry of AIDS. Journal of Clinical Psychiatry, 50, 9-14.

TYRer, P. (1992) Anxiety and deprescion: a clinical profile. In Experimental Approaches to Anxilety and Depression (eds J. M. Elliott, D. J. Heal \& C. A. Maraden), pp. 9-23. Chichester: Wiley. World HEaltr Organtzation (1992) The Tenth Revision of the International Classification of Diseases and Related Health Problems (ICD-10). Geneva: WHO.

Professor Peter Tyrer, FRCPsych, Academic Unit of Psychiatry, Imperial College School of Medicine at St. Mary's, St Charles' Hospital, London W10 6DZ

(Received and accepted 25 May 1995) 